Journal of Fundamental and Applied Sciences

ISSN 1112-9867

Available online at

http://www.jfas.info

\title{
OPTIMIZATION OF AEOLIAN ENERGY CONVERSION OPTIMISATION DE LA CONVERSION DE L'ENERGIE EOLIENNE
}

\author{
Y. Soufi", T. Bahi, M. F Harkat and M. Mohamedi \\ Département de génie électrique, Faculté des sciences de l'ingénieur, Université de \\ Tébessa
}

Received: 01 February 2010 / Accepted: 02 June 2010 / Published online: 30 June 2010

\begin{abstract}
The use of renewable energy increases, because people are increasingly concerned with environmental issues. Among renewable, wind power is now widely used. Their study showed that a value of wind speed, there is a maximum mechanical power supplied by the turbine. So, power is supplied are particularly changes with maximum speed.

However, the objective of this paper is to present an algorithm for optimal conversion of wind energy based on a criterion optimization that must maintain specific speed of the turbine at optimum speed which corresponds to the maximum power provided by the steady wind turbine. To this end, the object is to preserve the position of any static operating point on the characteristic of optimal.

To validate the model and algorithm for optimal conversion of wind energy, a series of numerical simulations carried out using the software MatLab Simulink will be presented is discussed.
\end{abstract}

Keywords: Renewable Energy System, Modelling, Synchronous Generator, Simulation.

Author Correspondence, e-mail: y_soufi@yahoo.fr

ICID: 1040442 


\section{INTRODUCTION}

Les énergies renouvelables permettent de produire de l'électricité proprement .Alors, les éoliens même si elles sont petites (jusqu'à $100 \mathrm{KW}$ ) ont des coûts de production très compétitifs. Ces dernières sont présentes dans le domaine l'électrification rurale pour une consommation locale d'électricité ou pour sa transformation vers d'autres vecteurs énergétiques. Cependant, ces petites éoliennes en milieu urbain sont des produits particulièrement nouveaux sur le marché. Les installations et les connexions au réseau sont également assez limitées. Par conséquent, pour aider au développement de ce marché, un effort de communication et d'accès à l'information doit être fait pris sérieusement en considération pour bien maitriser la technologie et les rendre en conséquent à des prix très abordables [1] [2].

Dans ce travail, nous nous sommes intéressés principalement aux éoliennes de petites puissances, en vue d'optimisation l'efficacité énergétique en utilisant des dispositifs d'électronique de puissance pour le contrôle de la puissance éolienne. L'objectif étant de présenter un algorithme permettant une conversion optimale de l'énergie. A cette fin, dans un premier temps, nous abordons plus spécifiquement la question de la chaîne, ainsi que la structure et les méthodes de contrôle-commande utilisées pour maximiser son efficacité énergétique. Puis, nous abordons plus particulièrement la méthode d'optimisation. L'objectif final est de disposer d'une modélisation à la fois suffisamment précise pour rendre compte des transferts énergétiques et suffisamment rapide pour permettre une optimisation du dimensionnement et/ou de la gestion du système. Ainsi, un critère d'optimisation qui doit maintenir la vitesse spécifique de l'éolienne à une vitesse optimale qui correspond à la puissance maximale fournie par l'aérogénérateur en régime stationnaire a été développé. Nous présentons des modèles de comportement, qui caractérisent le fonctionnement temporel de la chaîne éolienne. Pour cela, un modèle de la chaîne éolienne associe au modèle d'une génératrice synchrone avec un pont de diodes triphasé est considéré. En fin, le problème d'optimisation globale de la chaîne éolienne passive sera évoqué et les résultats seront analysés.

\section{Chaîne de conversion et modélisation}

L'énergie éolienne a connu une très forte croissance grâce aux avantages qu'elle présente pour l'environnement, aux percées technologique connexes et aux programmes 
d'encouragement gouvernementaux dans le monde [3] [4]. Les énergies renouvelables sont des énergies inépuisables. La plupart est fournie par le soleil, le vent, la chaleur de la terre, les chutes d'eau, les marées ou encore la croissance des végétaux, leur exploitation n'engendre pas ou peu de déchets et d'émissions polluantes. Ce sont les énergies de l'avenir grâce aux avantages qu'elles présentent pour l'environnement [5]. Les chaînes éoliennes produisent de l'électricité par aérogénérateurs. Elles se distinguent par leur puissance et leur type de fonctionnement (vitesse fixe ou variable). Cependant, pour optimiser le système de conversion d'énergie, il est nécessaire de connaître les différents organes de la chaîne . La figure 1 rappelle le schéma synoptique d'une chaîne éolienne de petite puissance. Celle-ci est constituée d'une voilure couplée directement à une génératrice synchrone qui débite sur un bus continu via un redresseur à diodes.

\section{Génératrice synchrone}

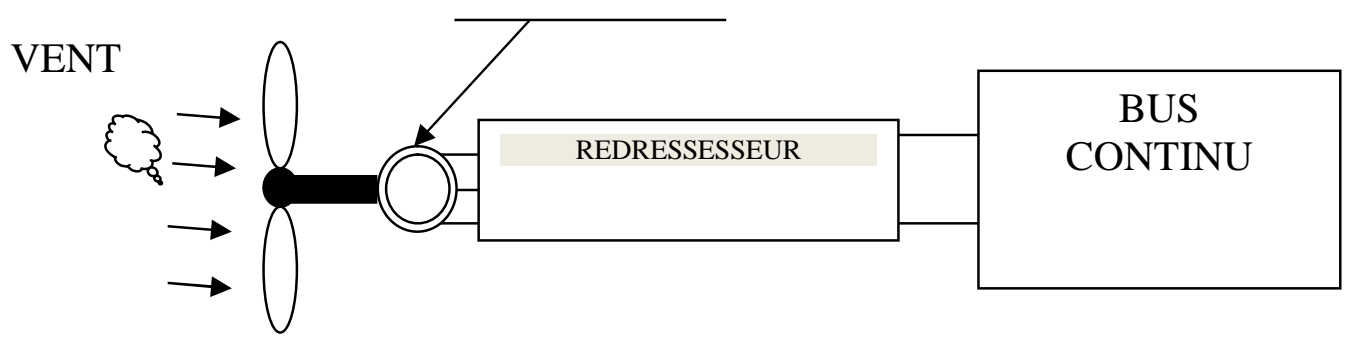

Fig.1. Chaîne Eolienne

L'énergie cinétique d'une masse d'air qui se déplace avec une vitesse v est :

$$
E_{c}=\frac{1}{2} \cdot m \cdot v^{2}
$$

Si cette énergie pouvait être complètement récupérée à l'aide d'une hélice qui balaie une surface $\mathrm{S}$ située perpendiculairement à la direction du vent, la puissance instantanée fournie par une turbine s'exprime par [6]:

$$
P_{v}=\frac{1}{2} \cdot \rho \cdot S \cdot V^{2}
$$

Cette conversion extraire une puissance inférieure $\mathrm{P}_{\mathrm{e}}$ à la puissance disponible $\mathrm{P}_{\mathrm{v}}$, on définit alors le coefficient de puissance par :

$$
C_{p}=\frac{P_{e}}{P_{v}} \quad ;\left(C_{p}\langle 1)\right.
$$

La puissance récupérée est donc donnée par : 


$$
P_{e}=0.5 \cdot \rho \cdot S \cdot C_{p} \cdot v^{3}
$$

Où :

$C_{p}$ : le coefficient de puissance une limite dite de Betz, $C_{p}$ limite $=0.59$.

$\rho$ : masse volumique de l'air (considéré égale à $1,25 \mathrm{~kg} / \mathrm{m}^{3}$ ),

$S$ : surface active de l'éolienne

$V$ : vitesse du vent.

Une turbine est typiquement caractérisée par sa courbe $C p=f(\lambda)$, avec $\lambda$ est le coefficient de vitesse réduite exprimé par la relation suivante:

$$
\lambda=\frac{\Omega \cdot R}{v}
$$

Où :

- $\quad$ est la vitesse angulaire de rotation

- $\quad$ R est le rayon de la pale

Le dimensionnement en puissance de l'ensemble de la turbine, du générateur et de toute la mécanique de structure (nacelle, mât) associée est défini pour une vitesse du vent nominale au-delà de laquelle il est nécessaire d'écrêter la puissance [7]. Ainsi, la courbe idéale et typique d'un aérogénérateur, a l'allure de celle présentée à la Figure 2. Elle comporte trois zones distinctes:

- la zone $I$, où $P_{\mathrm{e}}=0$ (la turbine ne fournit pas de puissance) ;

- la zone $I I$, dans laquelle la puissance fournie sur l'arbre dépend de la vitesse du vent $V$; - la zone $I I I$, où généralement la vitesse de rotation est maintenue constante par un dispositif de régulation et où la puissance $P_{\mathrm{e}}$ fournie reste sensiblement égale à la puissance nominale.

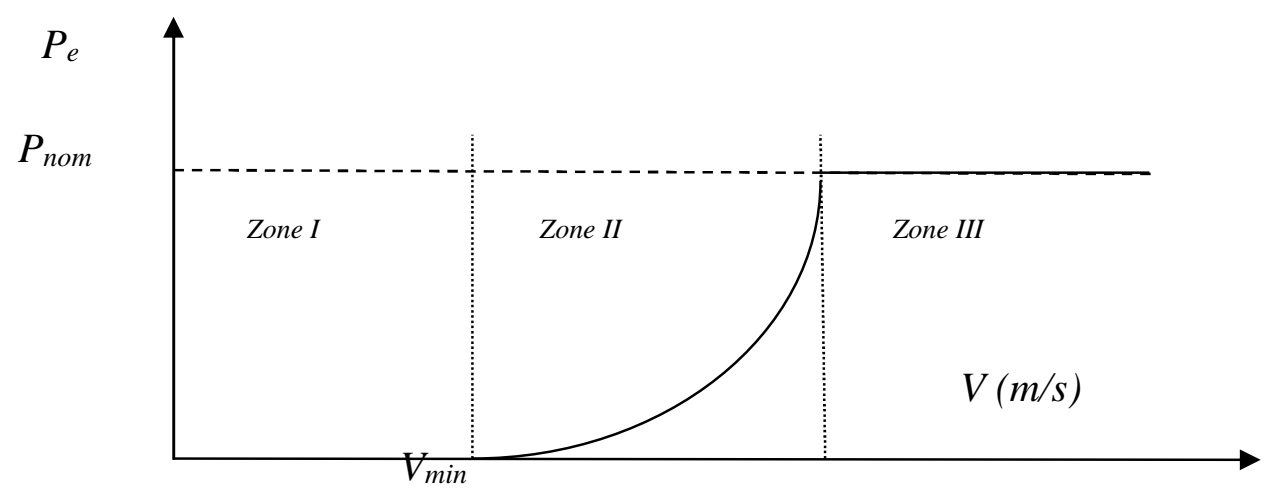

Fig.2. Puissance en fonction de la vitesse du vent. 
Les propriétés structurelles et dynamiques de la chaîne de transmission sont à considérer dans la phase de conception car elles affectent notablement la qualité de l'énergie électrique générée, au moins dans une gamme de fréquence harmonique intermédiaire. Cependant, la représentation mécanique de la chaîne éolienne tout entière est très complexe. Les éléments mécaniques de l'aérogénérateur et les forces subies ou transmises à travers ces éléments sont nombreux. Il faut par conséquent faire un choix des éléments et des grandeurs liées à ces éléments que l'on souhaite intégrer dans le modèle. Dans notre travail, nous avons adopté un modèle qui caractérise le comportement mécanique de la chaîne dans son ensemble [8] [9]. L'équation différentielle qui caractérise le comportement mécanique de l'ensemble turbine et génératrice est donnée par :

$$
J_{T} \frac{d \Omega}{d t}=C_{\text {eolienne }}-C_{e m}-f_{T}
$$

Avec :

$J_{T}$ : sont respectivement les inerties de la machine et de la turbine ;

$f_{T}$ :sont respectivement les coefficient de frottement de la machine et des pâles ;

$C_{\text {eolienne }}$ : le couple statique fournie par l'éolienne.

La figure 3, montre que la position du maximum de la courbe puissance en fonction de la vitesse de rotation change avec la vitesse du vent. Le réglage direct ou indirect de vitesse est nécessaire pour bien optimiser les transferts énergétiques.

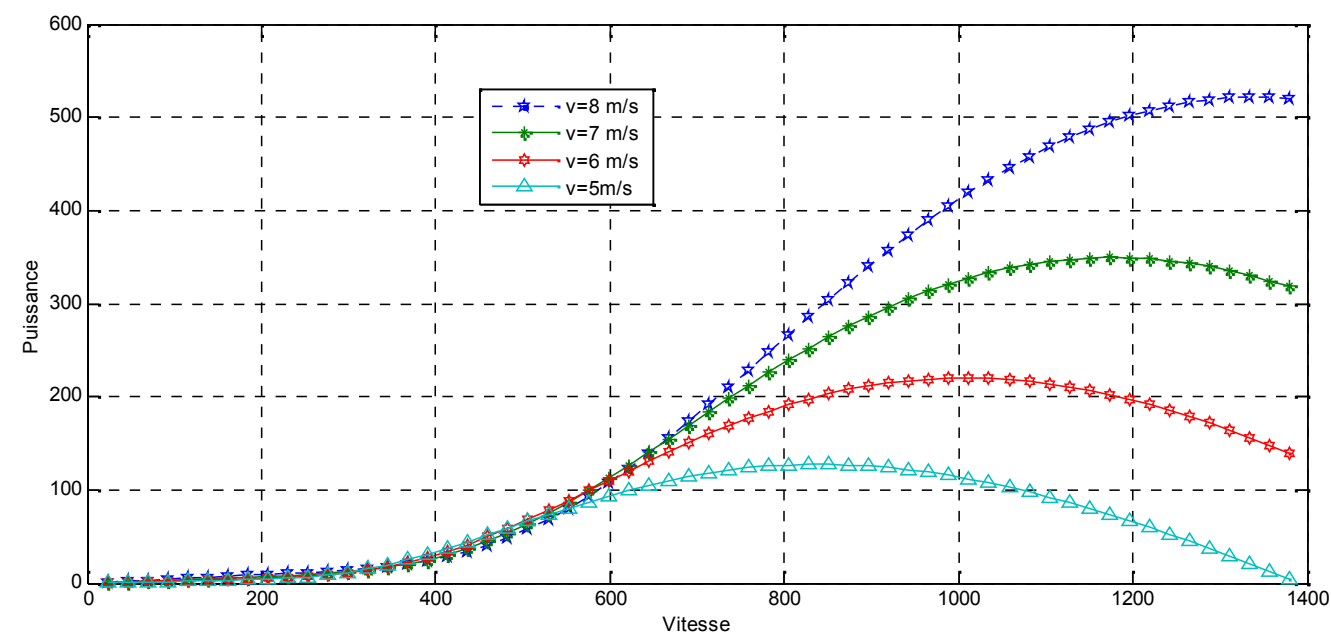

Fig.3. variation de la puissance éolienne en fonction de la vitesse du vent 
La génératrice synchrone est modélisée dans un repère abc. Le modèle circuit en termes de tension et de courant sous forme matricielle est donné par :

$$
[v]=[e]-\left[R_{s}\right][i]-\left[L_{s}\right] \frac{d[i]}{d t}
$$

Où,

$$
\begin{aligned}
& {[v]=\left[\begin{array}{lll}
v_{a} & v_{b} & v_{c}
\end{array}\right]^{t}} \\
& {[e]=\left[\begin{array}{lll}
\sin (Q) & \sin (Q-2 \pi / 3) & \sin (Q-4 \pi / 3)
\end{array}\right]^{t}}
\end{aligned}
$$

Avec :

$$
\begin{gathered}
Q=p \cdot \Omega_{t} \\
{\left[R_{s}\right]=\left[\begin{array}{ccc}
R_{s} & 0 & 0 \\
0 & R_{s} & 0 \\
0 & 0 & R_{s}
\end{array}\right] ; \quad\left[L_{s}\right]=\left[\begin{array}{ccc}
L_{s} & M_{s} & M_{s} \\
M_{s} & L_{s} & M_{s} \\
M_{s} & M_{s} & L_{s}
\end{array}\right]}
\end{gathered}
$$

L'expression du couple électromagnétique est donnée par :

$$
C_{e m}(t)=\frac{\sum_{i=a, b, c} e_{i}(t) \cdot i_{i}(t)}{\Omega(t)}
$$

Où, $V_{a}, V_{b}$, et $V_{c}$ sont les valeurs instantanées des tensions triphasées ;

Et, $i_{a}, i_{b}, i_{c}$ sont les valeurs instantanées des courants triphasés.

\section{OPTIMISATION ET RESULTATS}

La figure ci-dessous nous montre le bilan de puissance de la chaîne éolienne qui est caractérisé par deux rendements lié d'une part à l'extraction de l'énergie du vent (rendement aérodynamique, $\mathrm{P}_{\text {eolienne }} / \mathrm{P}_{\text {optimale}}$ ) et d'autre part lié aux pertes globales dans le système (génératrice + pont de diodes, $\mathrm{P}_{\text {utile }} / \mathrm{P}_{\text {eolienne}}$ ) ainsi le rendement global de la chaîne étudié est égale (Puissance utile / puissance éolienne) [10]. 


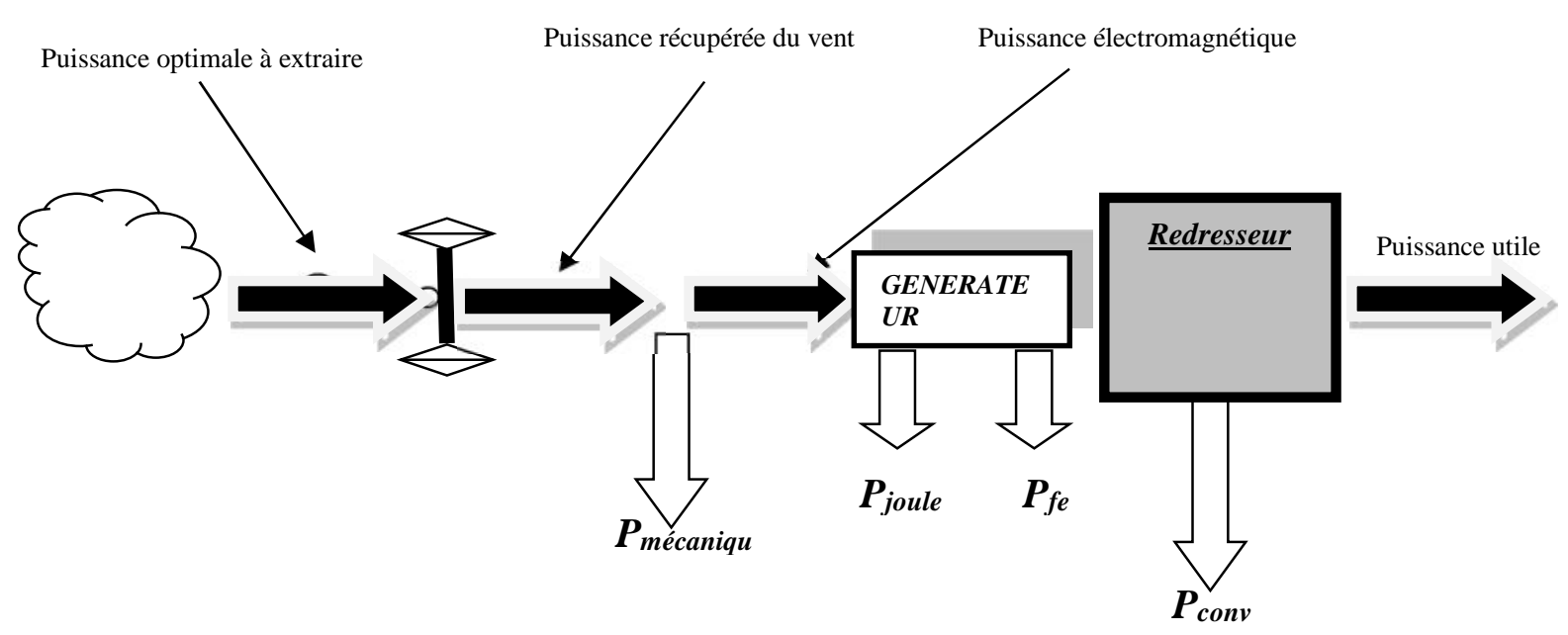

Fig.4. Bilan de puissance de la chaîne éolienne

Les pertes dans la génératrice sont subdivisées en pertes mécaniques, pertes Joules et fer.

\section{Pertes mécaniques}

Ces pertes sont dues aux frottements et dépendent de la vitesse de rotation de la génératrice. Elles sont données par la relation suivante:

$$
P_{m}=f_{m} \Omega^{2}
$$

\section{Pertes Joule}

Les pertes Joule sont causées par la résistance du bobinage statorique. Pour calculer ces pertes, nous avons utilisé la formule suivante :

$$
P_{j}=\sum_{i=a, b, c} R_{s} i_{s}
$$

Où, $i_{a}, i_{b}$ et $i_{c}$ sont les valeurs instantanées des courants triphasés.

\section{Pertes fer}

Ces pertes dépendent que de la fréquence et de la valeur maximale du flux. Elles sont désignés par $\mathrm{P}_{\text {fer }}$. La figure 5, illustre les rendements partiels de la chaîne éolienne $\left(\mathrm{P}_{\text {eol }}\right.$ / Popt, $\mathrm{P}_{\mathrm{u}} /$ Peol $)$ ainsi que le rendement global $\left(\mathrm{P}_{\mathrm{u}} / \mathrm{P}_{\mathrm{opt}}\right)$ de l'ensemble des solutions du front optimal. 


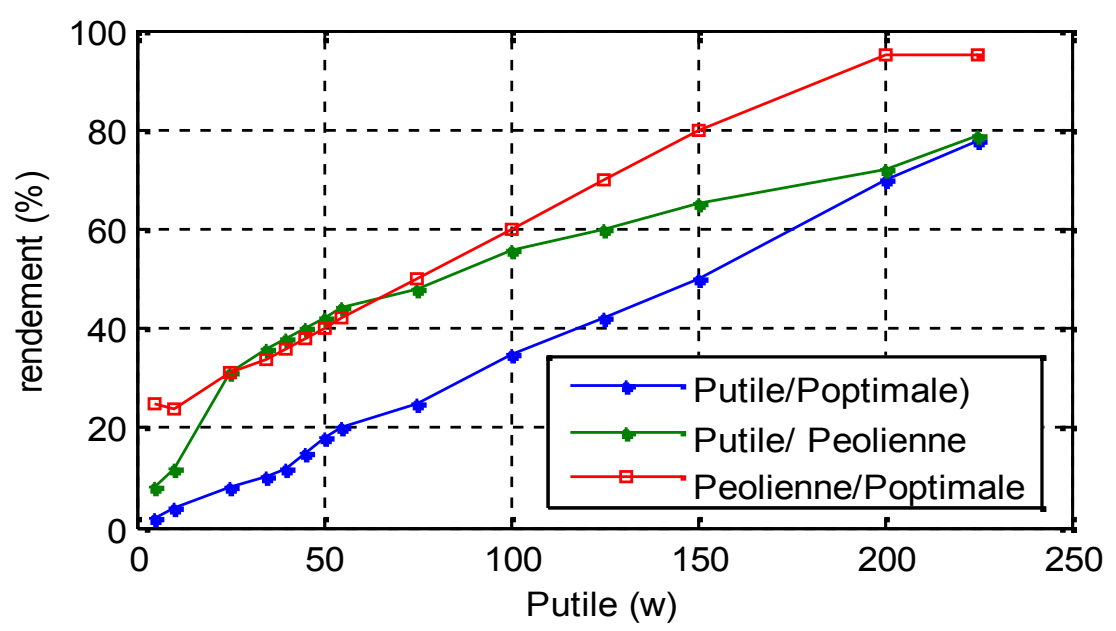

Fig.5. Rendement et puissances

Nous distinguons deux zones particulières la première correspond à la variation linéaire de la puissance éolienne extraite le long du front optimal. Dans deuxième zone,ou l'extraction de l'énergie du vent est quasiment stable et qui donne une puissance optimale.

Le rendement de l'ensemble du système varie d'une manière ascendante le long du front optimal. Le rendement maximal est de l'ordre de $85 \%$ par rapport à l'énergie extraite de l'énergie du vent. Le rendement maximal de la chaîne éolienne par rapport à la puissance optimale est satisfaisant.

\section{CONCLUSION}

Nous avons présenté la modélisation dynamique pour caractériser le comportement énergétique de la chaîne éolienne. L'optimisation de la chaîne éolienne a été réalisée et ses résultats analysés. La modélisation et la simulation ont été considérées afin de pouvoir intégrer les différents modèles dans un processus d'optimisation. A partir des résultats obtenus, nous concluons sur l'intérêt du dimensionnement par optimisation. Celui-ci a permis, d'extraction l'énergie de vent quasi optimale, et de réduire les pertes dans l'ensemble du système.

\section{REFERENCES}

[1] Multon B., Roboam X., Dakyo B., Nichita C., Gergaud O.et Ben Ahmed H. Aérogénérateurs électriques, Techniques de l'ingénieur, Ref D3960 - Vol D7, 2004. 
[2] Langlois O. (juin 2006). Conception d'un réseau de secours électrique pour l'aéronautique, Thèse de l'INP Toulouse.

[3] Maouedj R., Bousalm S., Hadji Y. and Benyoucef B. Application de l'énergie eolienne au pompage hydraulique au sud Algérien, 5 th international Conférence on Electrical Engineering 27-29 october 2008.

[4] Abdelhamid L., Abdessemed R. et Ammimeur H. Etude et Simulation des différents génératrices utilisées dans les systèmes éoliennes, 5 th international Conférence on Electrical Engineering 27-29 october 2008.

[5] Ackemann T ., Soder L. Renewable and sustainable energy Reviews. 2002, 6, 67-68.

[6] Munteanu I. (1997). Etude et simulation des aerogenerateurs: Applications a un aerogenerateur à courant continu, DEA instrumentation et commande, université de Havre.

[7] Abdelli A., Sareni B., Roboam X. Optimization of a Small Passive Wind Turbine Generator with Multi-objective Genetic Algorithms, OIPE 2006.

[8] Cardenas Dobson R. (1996). Control of Wind Turbine Using a Switched Reluctance Generator, These Doctorale, l'Université de Nottingham.

[9] Doudou Diop A. (1977) Contribution au développement d'un simulateur électromécanique d'aerogenerateur: simulation et commande en temps réel d'une turbine de puissance moyenne a angle de calage variable, thèse de doctorat, université de Havre.

[10] Abdelli A. (2007). Optimisation multicritère d'une chaîne éolienne passive , thèse de doctorat, l'institut national polytechnique de Toulouse, France.

\section{How to cite this article}

Soufi Y, Bahi T, Harkat M F, Mohamedi M. Optimization of aeolian energy conversion optimisation de la conversion de l'energie eolienne. J Fundam Appl Sci. 2010, 2(1), 183-191. 